\title{
A REMARK ON A CONJECTURE OF BUZZARD-GEE AND THE COHOMOLOGY OF SHIMURA VARIETIES
}

\author{
Christian JOHANSSON
}

\begin{abstract}
We compare the conjecture of Buzzard-Gee on the association of Galois representations to $C$-algebraic automorphic representations with the conjectural description of the cohomology of Shimura varieties due to Kottwitz, and the reciprocity law at infinity due to Arthur. This is done by extending Langlands's representation of the $L$-group associated with a Shimura datum to a representation of the $C$-group of Buzzard-Gee. The approach offers an explanation of the explicit Tate twist appearing in Kottwitz's description.
\end{abstract}

\section{Introduction}

A central theme in modern algebraic number theory is the connection between automorphic forms and Galois representations. This link has its origins in class field theory, and evolved from the work of Weil et al. among others before its scope was vastly expanded by Langlands in the late 1960s and 1970s, linking the emerging theory of automorphic representations to Grothendieck's conjectural theory of motives, where Galois representations appear as realizations. For automorphic representations of $\mathrm{GL}_{n}$, the first precise conjectures were given by Clozel in [Clo] where he defined a notion of algebraicity and conjectured that there should be motives (hence Galois representations) associated with algebraic automorphic representations.

In [BG], Buzzard and Gee generalize the notion of algebraicity and (a weak form of) Clozel's conjectures to an arbitrary connected reductive group $G$ over a number field $F$. Perhaps somewhat surprisingly, they define two notions of algebraicity. On the one hand they define a notion of $L$-algebraicity, and given an $L$-algebraic automorphic representation $\pi$ of $G$ they conjecture the existence of an $\ell$-adic Galois representation

$$
\rho_{\pi}^{L}: \operatorname{Gal}(\bar{F} / F) \rightarrow{ }^{L} G\left(\overline{\mathbb{Q}}_{\ell}\right)
$$

They also define a notion of $C$-algebraicity which generalizes Clozel's definition of algebraicity. Buzzard and Gee (following an idea of Deligne) define a canonical $\mathbb{G}_{m}$-extension $\widetilde{G}$ of $G$ and given a $C$-algebraic automorphic representation $\pi$ of $G$, they canonically construct an $L$-algebraic automorphic representation $\widetilde{\pi}$ of $\widetilde{G}$ and thus conjecturally get an associated Galois representation

$$
\rho_{\pi}=\rho_{\widetilde{\pi}}^{L}: \operatorname{Gal}(\bar{F} / F) \rightarrow{ }^{L} \widetilde{G}\left(\overline{\mathbb{Q}}_{\ell}\right)={ }^{C} G\left(\overline{\mathbb{Q}}_{\ell}\right)
$$

Since the beginnings of the theory, an important test case for any conjecture on the association of Galois representation to automorphic representations has been the case of Shimura varieties, starting with the modular curve. Langlands initiated a program to compute the ( $\ell$-adic) cohomology of arbitrary Shimura varieties (with coefficients in

Received by the editors July 31, 2012. 
certain local systems) and later on Kottwitz gave a very precise conjectural description of these cohomology groups [Kot]. The automorphic representations that contribute to this description are called cohomological and they are the one of most important classes of $C$-algebraic automorphic representations.

In this short paper, we wish to test the Buzzard-Gee conjecture on the association of Galois representations with $C$-algebraic automorphic forms in the case of cohomological automorphic representations on groups admitting a Shimura variety. Namely, given a cohomological automorphic representation $\pi$, we show that Kottwitz's description implies (or rather is equivalent to) that the composition of $\rho_{\pi}$ with a certain fixed algebraic representation $r_{C}$ of ${ }^{C} G$ occurs in the cohomology (for the precise statement, see Conjecture 8). The representation $r_{C}$ is an extension to ${ }^{C} G$ of a representation of ${ }^{L} G$ (originally defined by Langlands in [Lan]) that is used in Kottwitz's description. Our minor reformulation has the slight advantage that it explains (or perhaps internalizes) a somewhat mysterious Tate twist by half the dimension of the Shimura variety that occurs in Kottwitz's description.

The structure of this note is as follows: In Section 2 we define the representation $r_{C}$ and in Section 3 we prove Corollary 3 which is the main result needed to relate the Buzzard-Gee conjecture and Kottwitz's description of the cohomology of Shimura varieties. This uses only some basic Lie theory and the definition of a Shimura datum. Finally in Section 4, we recall Kottwitz's description together with a related result of Arthur [Art] and make the comparison.

\section{The representation $r_{C}$}

In this section, we will set up some notation, recall the notion of a $C$-group from [BG] and define a representation $r_{C}$ which will be an extension of a representation of Langlands that we will denote $r_{L}$ (whose definition we will recall below). For simplicity, all our dual groups and $L$-groups will be complex algebraic groups and complex pro-algebraic groups, respectively, and will be identified with their $\mathbb{C}$-points. In Section 4 we will fix an isomorphism $\iota: \mathbb{C} \cong \overline{\mathbb{Q}}_{\ell}$, where $\ell$ is a fixed rational prime. Given a connective reductive group $G$ over a field $F$ with $L$-group ${ }^{L} G=\operatorname{Gal}(\bar{F} / F) \ltimes \widehat{G}$ (we will always use the Galois form of the $L$-group), we may use $\iota$ to consider the associated pro-algebraic group ${ }^{L} G\left(\overline{\mathbb{Q}}_{\ell}\right)$ over $\overline{\mathbb{Q}}_{\ell}$, identified with its $\overline{\mathbb{Q}}_{\ell}$-points, and by abuse of notation we will denote by $\iota$ the map ${ }^{L} G \rightarrow{ }^{L} G\left(\overline{\mathbb{Q}}_{\ell}\right)$ induced by $\iota$.

Let $G$ be a connected reductive group over $\mathbb{Q}$ and let $X$ be a $G(\mathbb{R})$-conjugacy class of homomorphisms

$$
h: \mathbb{S} \rightarrow G_{\mathbb{R}}
$$

of real algebraic groups, where $\mathbb{S}$ denotes Deligne's torus $\operatorname{Res}_{\mathbb{R}}^{\mathbb{C}} \mathrm{GL}_{1 / \mathbb{C}}$. The pair $(G, X)$ is required to satisfy Deligne's axioms for a Shimura datum (see [Del] Section 2.1.1). We will let $E$ denote the reflex field of $(G, X)$ and we denote by $d$ the complex dimension of $X$. Given $h \in X$, we let $\mu_{h}$ denote the corresponding cocharacter of $G_{\mathbb{C}}$ defined by

$$
\mu_{h}(z)=h_{\mathbb{C}}(z, 1)
$$

where we have fixed an isomorphism $\mathbb{S}(\mathbb{C}) \cong \mathbb{C}^{\times} \times \mathbb{C}^{\times}$(and $\mathbb{S}(\mathbb{R})=\mathbb{C}^{\times}$embeds by $z \mapsto(z, \bar{z}))$. We will follow the conventions of [Del] for associating real Hodge 
structures with representations of $\mathbb{S}$ (i.e., the $(p, q)$-space is the subspace where $\mathbb{S}(\mathbb{C})$ acts by $\left.(z, w) \mapsto z^{-p} w^{-q}\right)$ and the normalization of the reciprocity map of local class field theory (uniformizers go to geometric Frobenii). Fix a pinning $(T, B)$ of $G$ with corresponding pinning $(\widehat{T}, \widehat{B})$ of $\widehat{G}$ that is fixed by the Galois action (we suppress the choice of elements in the root spaces for the simple roots). This gives us the notion of dominant weights and coweights and positive roots and coroots for $G$ and $\widehat{G}$. We let $\mu$ denote an element of the conjugacy class of $\left(-\mu_{h}\right)_{h \in X}$ which is antidominant. The representation $r_{L}$ is defined in two steps. $\mu$ defines an antidominant integral weight $\widehat{\mu}$ of $\widehat{G}$ and hence a unique irreducible representation

$$
r: \widehat{G} \rightarrow \operatorname{Aut}\left(V_{\mu}\right)
$$

that has $\widehat{\mu}$ as an extreme weight. We extend this to our desired representation

$$
r_{L}:{ }^{L} G_{E}=\Gamma_{E} \ltimes \widehat{G} \rightarrow \operatorname{Aut}\left(V_{\mu}\right)
$$

by letting $\Gamma_{E}=\operatorname{Gal}(\overline{\mathbb{Q}} / E)$ act trivially on the weight space of $\widehat{\mu}$.

Buzzard and Gee have defined the notion of a $C$-group ${ }^{C} G$ for $G$ ([BG] Definition 5.3.2), which is defined to be the $L$-group ${ }^{L} \widetilde{G}$ of a canonical extension

$$
1 \rightarrow \mathrm{GL}_{1} \rightarrow \widetilde{\mathrm{G}} \rightarrow \mathrm{G} \rightarrow 1
$$

of $G$. Proposition 5.3 .3 of $[\mathrm{BG}]$ gives a central isogeny

$$
\widehat{G} \times \mathbb{C}^{\times} \rightarrow \widehat{\widetilde{G}}
$$

which is Galois-equivariant and whose kernel is generated by the element $(e,-1)$ of order 2. Here, $e=\widehat{\chi}(-1)$, where $\chi$ is the sum of positive roots of $G$ and $\widehat{\chi}$ is the sum of the positive coroots of $\widehat{G}$. We wish to extend $r_{L}$ to a representation $r_{C}$ of ${ }^{C} G_{E}=\Gamma_{E} \ltimes \widehat{\widetilde{G}}$. Let $\langle-,-\rangle$ denote the pairing between the character and cocharacter lattices of $G$ and $\widehat{G}$ to $\mathbb{Z}$. Part 1 ) of the following Lemma is well known.

Lemma 1. (1) $\langle\chi, \mu\rangle=-d$.

(2) e acts as $(-1)^{-d}$ on $V_{\mu}$.

Proof. (1) By axiom (2.1.1.1) for a Shimura datum (in the notation of [Del] Section 2.1.1) and the choice of $\mu, \mu(z)$ acts as $z^{-1}$ or 1 on the positive root spaces (i.e., $\langle\alpha, \mu\rangle=-1$ or 0 for each positive root $\alpha$ ) and as $z$ or 1 on the negative root spaces. The result follows since there are $d$ roots on which $\mu(z)$ acts as $z^{-1}$, by axiom (2.1.1.2) for a Shimura datum ([Del] Section 2.1.1).

(2) Since $e$ is central it acts on $V_{\mu}$ by a scalar and hence it is enough to compute the action on the weight space of $\widehat{\mu}$. By part 1 ), the action is by

$$
\widehat{\mu}(e)=\widehat{\mu}(\widehat{\chi}(-1))=(-1)^{\langle\widehat{\mu}, \widehat{\chi}\rangle}=(-1)^{\langle\chi, \mu\rangle}=(-1)^{-d} .
$$

Let us now extend $r$ to a representation of $\widehat{G} \times \mathrm{GL}_{1}$ by

$$
(g, z) \mapsto r(g) z^{-d} .
$$

By Lemma $1(e,-1)$ acts trivially so this defines a representation of $\widehat{\widetilde{G}}$. We may extend this to our desired representation $r_{C}$ of ${ }^{C} G_{E}$ on $V_{\mu}$ by letting $\Gamma_{E}$ act trivially on the 
weight space of $\widehat{\mu}$; by the $\Gamma_{E}$-equivariance of the isogeny $\widehat{G} \times \mathrm{GL}_{1} \rightarrow \widehat{\widetilde{G}}$ this defines an extension of $r_{L}$.

\section{The result}

Let $\tau$ be an irreducible algebraic representation of $G$, and let $\pi$ be a discrete automorphic representation of $G$ which is cohomological for $\tau$, i.e., there is an integer $i$ such that $H^{i}\left(\mathfrak{g}_{\infty}, K_{\infty}, \pi_{\infty} \otimes \tau\right) \neq 0$, where $\mathfrak{g}_{\infty}$ is the Lie algebra of $G_{\mathbb{R}}, K_{\infty} \subseteq G(\mathbb{R})$ is the stabilizer of some fixed $x \in X$ and $H^{i}\left(\mathfrak{g}_{\infty}, K_{\infty},-\right)$ is the relative Lie algebra cohomology. Lemma 7.2.2 of [BG] says that $\pi$ is $C$-algebraic ([BG], Definition 3.1.2).

Remark. $\pi$ is also $C$-arithmetic ([BG], Definition 3.1.4). This follows from the argument in Section 2.3 of $[\mathrm{BR}]$ taking coefficients in $\mathcal{F}_{\tau}$ (defined near the end of the first paragraph of Section 4 of this paper) instead of the trivial local system.

Let $\pi$ be a $C$-algebraic automorphic representation on $G$. We will briefly review the construction of an $L$-algebraic ([BG], Definition 3.1.1) automorphic representation $\tilde{\pi}$ of $\widetilde{G}$, canonically associated to $\pi$ (see [BG], discussion before Conjecture 5.3.4). Given $\pi$, one uses the canonical map $\widetilde{G} \rightarrow G$ to pull back $\pi$ to a $C$-algebraic automorphic representation $\pi^{\prime}$ ([BG] Lemma 5.1.2). The central isogeny $\widehat{G} \times \mathrm{GL}_{1} \rightarrow \widehat{\widetilde{G}}$ mentioned in the previous section is dual to an isogeny $(c, \xi): \widetilde{G} \rightarrow G \times \mathrm{GL}_{1}$ defined over $\mathbb{Q}$. Then

$$
g \mapsto|\xi(g)|^{1 / 2}
$$

is a character $\widetilde{G}(\mathbb{A}) \rightarrow \mathbb{C}^{\times}$, and we define $\widetilde{\pi}$ to be the twist $\pi^{\prime} \otimes|\xi(-)|^{1 / 2}$. It is an $L$-algebraic automorphic representation of $\widetilde{G}$.

For a local or global field $F$ of characteristic 0 we let $\mathcal{L}_{F}$ denote the Langlands group of $F$. It carries a canonical surjection $\mathcal{L}_{F} \rightarrow W_{F}$, where $W_{F}$ is the Weil group of $F$. When $F$ is local, we have $\mathcal{L}_{F}=W_{F}$ if $F$ is archimedean (with the canonical map being the identity) and we take $\mathcal{L}_{F}=W_{F} \times \mathrm{SL}_{2}(\mathbb{C})$ if $F$ is non-archimedean (the canonical map being projection onto the first factor). When $F$ is global, this group only exists conjecturally. We will use it only for motivation (in particular to make the comparison with [Kot]); in the end all conjectures and results may be stated using only $\mathcal{L}_{F}$ for local fields.

Let $|-|$ denote the composition of $\mathcal{L}_{F} \rightarrow W_{F}$ with the norm character $W_{F} \rightarrow \mathbb{C}^{\times}$.

Lemma 2. 1) Let $p$ be a finite prime where $\pi$ and $G$ are unramified and let $\phi_{p}$ : $\mathcal{L}_{\mathbb{Q}_{p}} \rightarrow{ }^{L} G$ be the L-parameter (Satake parameter) associated with $\pi_{p}$. Then the $L$-parameter $\widetilde{\phi}_{p}: \mathcal{L}_{\mathbb{Q}_{p}} \rightarrow{ }^{C} G$ attached to $\tilde{\pi}_{p}$ is given by

$$
\widetilde{\phi}_{p}(w)=\left(\phi_{p}(w),|w|^{1 / 2}\right)
$$

where we abuse notation and denote by $\left(\phi_{p}(w),|w|^{1 / 2}\right)$ the image of $\left(\phi_{p}(w),|w|^{1 / 2}\right) \in$ ${ }^{L} G \times \mathbb{C}^{\times}$in ${ }^{C} G$.

2) Let $\phi_{\mathbb{C}}: \mathcal{L}_{\mathbb{C}} \rightarrow{ }^{L} G$ be the restriction of the $L$-parameter associated with $\pi_{\infty}$. Then the restriction of the L-parameter of $\tilde{\pi}_{\infty}$ to $\mathcal{L}_{\mathbb{C}}$ is given by

$$
\widetilde{\phi}_{\mathbb{C}}(w)=\left(\phi_{\mathbb{C}}(w),|w|^{1 / 2}\right) .
$$




\section{Proof.}

(1) This follows from the construction of the unramified Local Langlands correspondence as described in Section 10.4 of [Bor] and is implicit in [BG] (in the derivation of Conjecture 5.3.4 from Conjecture 3.2.1); we will content ourselves with giving a brief sketch of the proof. Let $\widetilde{T}$ resp. $\widetilde{B}$ be the inverse images of $T$ resp. $B$ under $\widetilde{G} \rightarrow G$. $\pi_{p}$ occurs as a subquotient of some unramified principal series $\operatorname{Ind}_{B}^{G} \chi$ (normalized induction), where $\chi: T\left(\mathbb{Q}_{p}\right) \rightarrow \mathbb{C}^{\times}$is an unramified character. Since parabolic induction behaves well with respect to pullback $\widetilde{G}\left(\mathbb{Q}_{p}\right) \rightarrow G\left(\mathbb{Q}_{p}\right), \pi^{\prime}$ occurs as a subquotient of Ind $\widetilde{\widetilde{G}} \chi^{\prime}$, where $\chi^{\prime}$ is the composition of $\chi$ with $\widetilde{T} \rightarrow T$. This implies that the $L$-parameter of $\pi^{\prime}$ is $w \mapsto\left(\phi_{p}(w), 1\right)$ since the correspondence for unramified characters of unramified tori is functorial. To get the $L$-parameter of $\tilde{\pi}$ we use that the unramified Local Langlands correspondence behaves well with respect to unramified twists; see e.g., Remark 2.2.1 of [BG] (although it is perhaps simpler to deduce this from the construction in [Bor] since parabolic induction behaves well under twists, rather than using the Satake isomorphism directly as is done in $[\mathrm{BG}])$.

(2) This is proved in exactly the same way as (1), although the details are simpler, using the construction of the complex Local Langlands correspondence (see [Bor] Section 11.4). Here one deduces the behavior with respect to twists from same property for parabolic induction as indicated in the end of the proof of (1).

The following simple consequence is our main technical result.

Corollary 3. With notation as in Lemma 2, we have

$$
\left(r_{L} \circ \phi_{v}\right) \otimes|-|^{-d / 2}=r_{C} \circ \widetilde{\phi}_{v}
$$

for $v$ finite where $\pi$ and $G$ are unramified or $v=\mathbb{C}$.

Proof. By Lemma 2 and the definition of $r_{C}$ we have

$$
r_{C}\left(\widetilde{\phi}_{v}(w)\right)=r_{C}\left(\phi_{v}(w),|w|^{1 / 2}\right)=r_{L}\left(\phi_{v}(w)\right)|w|^{-d / 2}
$$

as desired.

\section{Comparison with Kottwitz's Conjecture and Arthur's Result}

Let us try to briefly describe the conjecture of Kottwitz and the result of Arthur. For simplicity, we will follow Arthur's presentation in Section 9 of [Art]. Recall our Shimura datum $(G, X)$. If $K \subseteq G\left(\mathbb{A}^{\infty}\right)$ is a compact open subgroup we will let $S h_{K}$ denote the canonical model over $E$ of the corresponding Shimura variety and $\overline{S h}_{K}$ its minimal compactification [Pin]. We write $S h$ for the inverse system $\left(S h_{K}\right)_{K}$ and $\overline{S h}$ for $\left(\overline{S h}_{K}\right)_{K}$. The reflex field $E$ comes with an embedding into $\mathbb{C}$ and we let $\overline{\mathbb{Q}}$ denote the algebraic closure of $\mathbb{Q}$ inside $\mathbb{C}$. Let $\ell$ be a fixed rational prime; we fix an isomorphism $\iota: \overline{\mathbb{Q}}_{\ell} \cong \mathbb{C}$, thus we may view $\overline{\mathbb{Q}}$ as a subfield of $\overline{\mathbb{Q}}_{\ell}$. Let $\tau$ be an algebraic representation of $G$. Then we may associate to $\tau$ a sheaf $\mathcal{F}_{\tau}$ which is either a constructible local system of $\mathbb{Q}$-vector spaces on $S h_{K}(\mathbb{C})^{\text {an }}$, a smooth $\overline{\mathbb{Q}}_{\ell^{-}}$-sheaf on $S h_{K}$ 
or a vector bundle with flat connection on $S h_{K}$. They satisfy various compatibilities with respect to transition maps in $S h$ and comparison theorems for the relevant cohomology theories; we will abuse notation and denote them all by $\mathcal{F}_{\tau}$, as well as the canonical extension of the smooth $\overline{\mathbb{Q}}_{\ell}$-sheaf $\mathcal{F}_{\tau}$ on $S h_{K}$ to $\overline{S h}_{K}$ (defined via the theory of perverse sheaves). There is a geometric action of $G\left(\mathbb{A}^{\infty}\right)$ on $S h$ and $\overline{S h}$. For any suitable cohomology theory $H$ we write

$$
\begin{gathered}
H^{*}\left(S h_{K}, \mathcal{F}_{\tau}\right)=\bigoplus_{i} H^{i}\left(S h_{K}, \mathcal{F}_{\tau}\right) \\
H^{*}\left(S h, \mathcal{F}_{\tau}\right)=\lim _{\rightarrow} H^{*}\left(S h_{K}, \mathcal{F}_{\tau}\right) \in \operatorname{Mod}\left(G\left(\mathbb{A}^{\infty}\right) \times ?\right)
\end{gathered}
$$

where the ? signifies that the cohomology theory may carry extra structure. We use analogous notation for $\overline{S h}$.

The starting point for the analysis of the cohomology of Shimura varieties is the result

$$
H_{(2)}^{*}\left(S h, \mathcal{F}_{\tau}\right)=\bigoplus_{\pi} m(\pi) \pi^{\infty} \otimes H^{*}\left(\mathfrak{g}_{\infty}, K_{\infty}, \pi_{\infty} \otimes \tau\right) \in \operatorname{Mod}\left(G\left(\mathbb{A}^{\infty}\right)\right)
$$

of Borel and Casselman [BC], where $H_{(2)}$ denotes $L^{2}$-cohomology, $\pi$ runs through the irreducible admissible representations of $G(\mathbb{A}), m(\pi)$ is the multiplicity of $\pi$ in the discrete spectrum of $L^{2}\left(G(\mathbb{Q}) \backslash G(\mathbb{A})^{1}\right)$ and $H^{*}\left(\mathfrak{g}_{\infty}, K_{\infty},-\right)=\bigoplus_{i} H^{i}\left(\mathfrak{g}_{\infty}, K_{\infty},-\right)$ is total relative Lie algebra cohomology. To $\pi$ such that $m(\pi) \neq 0$ one should conjecturally be able to attach a discrete $A$-parameter $\psi$ and we may, assuming Conjecture 8.1 of $[$ Art $]$, rewrite the above as

$$
H_{(2)}^{*}\left(S h, \mathcal{F}_{\tau}\right)=\bigoplus_{\psi} \bigoplus_{\pi \in \Pi_{\psi}} m_{\psi}(\pi) \pi^{\infty} \otimes H^{*}\left(\mathfrak{g}_{\infty}, K_{\infty}, \pi_{\infty} \otimes \tau\right)
$$

where $\psi$ runs through the set $\Psi_{0}(G)$ of discrete $A$-parameters ([Art] p. 56), $\Pi_{\psi}$ is the $A$-packet attached to $\psi$ and $m_{\psi}(\pi)$ is a certain multiplicity. Put

$$
V_{\psi}=\bigoplus_{\pi_{\infty} \in \Pi_{\psi_{\infty}}} H^{*}\left(\mathfrak{g}_{\infty}, K_{\infty}, \pi_{\infty} \otimes \tau\right)
$$

Associated with $\psi$ is a group $S_{\psi}$ defined on p. 52 of [Art] (as well as local versions $S_{\psi_{v}}$ for any place $v$ of $\left.\mathbb{Q}\right)$. $V_{\psi}$ carries a representation of $S_{\psi_{\infty}}$, a Hodge structure and a Lefschetz decomposition ([Art] pp. 59-61). Arthur then defines, for each $\pi$, a vector space $U_{\pi}$ that only depends on $\pi^{\infty}$ and carries an action of $S_{\psi}$, an action of $S_{\psi}$ on $U_{\pi} \otimes V_{\psi}$ and rewrites (4.1) as

$$
H_{(2)}^{*}\left(S h, \mathcal{F}_{\tau}\right)=\bigoplus_{\psi} \bigoplus_{\pi^{\infty} \in \Pi_{\psi} \infty} \pi^{\infty} \otimes\left(U_{\pi} \otimes V_{\psi}\right)_{\epsilon_{\psi}}
$$

where $\epsilon_{\psi}$ is a certain sign character of $S_{\psi}$ and $(-)_{\epsilon_{\psi}}$ denotes the subspace where $S_{\psi}$ acts as $\epsilon_{\psi}$. This uses equation (8.5) of [Art] for the multiplicity $m_{\psi}(\pi)$. By various comparison theorems together with Zucker's conjecture equation (4.2) holds for $\ell$-adic intersection cohomology of the minimal compactification

$$
H_{e t, \ell}^{*}\left(\overline{S h}, \mathcal{F}_{\tau}\right)=\bigoplus_{\psi} \bigoplus_{\pi^{\infty} \in \Pi_{\psi} \infty} \pi^{\infty} \otimes\left(U_{\pi} \otimes V_{\psi}\right)_{\epsilon_{\psi}}
$$


as $G\left(\mathbb{A}^{\infty}\right)$-modules, after applying $\iota$ (as $\iota$ is fixed, we will omit it from the notation). $\left(U_{\pi} \otimes V_{\psi}\right)_{\epsilon_{\psi}}$ carries an action of $S_{\psi}$ (by $\epsilon_{\psi}$ ), and for $L^{2}$-cohomology it carries a Hodge structure and a Lefschetz decomposition, whereas for $\ell$-adic intersection cohomology it carries a representation of $\operatorname{Gal}(\overline{\mathbb{Q}} / E)$ and a Lefschetz decomposition. We wish to describe this extra structure.

To that end, let $\psi: \mathcal{L}_{\mathbb{Q}} \times \mathrm{SL}_{2}(\mathbb{C}) \rightarrow{ }^{L} G$ be the $A$-parameter attached to $\pi$. We write $\psi_{E}$ for the restriction to $\mathcal{L}_{E} \times \mathrm{SL}_{2}(\mathbb{C})$ and let $\phi_{E}$ denote the associated $L$-parameter $\mathcal{L}_{E} \rightarrow{ }^{L} G$, defined by

$$
\phi_{E}(w)=\psi_{E}\left(w,\left(\begin{array}{cc}
|w|^{1 / 2} & 0 \\
0 & |w|^{-1 / 2}
\end{array}\right)\right) .
$$

Kottwitz composes $\phi_{E}$ with $r_{L}$ to obtain an $L$-parameter $\phi_{E, r_{L}}$ and conjectures that there should exist a motive $M_{\psi}$ over $E$ whose $\ell$-adic étale realization $H_{e t, \ell}\left(M_{\psi}\right)$ satisfies

$$
W D_{\iota}\left(\left.H_{e t, \ell}\left(M_{\psi}\right)\right|_{G a l\left(\bar{E}_{v} / E_{v}\right)}\right)=\left.\left(\phi_{E, r_{L}}|-|^{-d / 2}\right)\right|_{\mathcal{L}_{E_{v}}}
$$

for each place $v \nmid \ell \infty$ of $E$ (where $W D_{\iota}$ means take the associated complex WeilDeligne representation, using $\iota)$. Moreover, its de Rham realization $H_{d R, v}\left(M_{\psi}\right)$ with respect to a place $v \mid \infty$ of $E$ should satisfy

$$
H_{d R, v}\left(M_{\psi}\right)=\left.\left(\phi_{E, r_{L}}|-|^{-d / 2}\right)\right|_{\mathcal{L}_{\bar{E}_{v}}} .
$$

Here $H_{d R, v}\left(M_{\psi}\right)$, as a Hodge structure, carries an action of $\mathbb{C}^{\times} \cong W_{\bar{E}_{v}}$. In both cases the Lefschetz decomposition on $H_{e t}\left(M_{\psi}\right)$ and $H_{d R}\left(M_{\psi}\right)$ should be given by $\left.r_{L} \circ \psi_{E}\right|_{\mathrm{SL}_{2}(\mathbb{C})}$. Kottwitz verifies ([Kot] p. 200) that these actions of $r_{L} \circ \psi_{E}$ (hence of $\phi_{E, r_{L}}$ and its twist) commute with the action of $S_{\psi}$ on $V_{\psi}$, and hence that we get induced structures on $\left(U_{\pi} \otimes V_{\psi}\right)_{\epsilon_{\psi}}$. For the finite places we then have

Conjecture 4 (Kottwitz, [Kot] p. 201). Assume that the derived group $G^{\text {der }}$ of $G$ is simply connected and that the maximal $\mathbb{R}$-split torus of the center $Z(G)$ of $G$ is $\mathbb{Q}$-split. Then the $\operatorname{Gal}(\overline{\mathbb{Q}} / E)$-representation

$$
W_{e t, \ell}^{*}\left(\pi^{\infty}\right)=\operatorname{Hom}_{G\left(\mathbb{A}^{\infty}\right)}\left(\pi^{\infty}, H_{e t, \ell}^{*}\left(\overline{S h}, \mathcal{F}_{\tau}\right)\right)
$$

is isomorphic to

$$
\bigoplus_{\psi: \pi \infty \in \Pi_{\psi} \infty}\left(U_{\pi} \otimes H_{e t}\left(M_{\psi}\right)\right)_{\epsilon_{\psi}} .
$$

\section{Remark.}

(1) Kottwitz does much more than stating the conjecture. Assuming Arthur's conjectures on the discrete spectrum (i.e., Conjectures 8.1 and 8.5 of [Art]), some conjectures on transfer and a formula for the number of points modulo primes of good reduction for $S h$, Kottwitz computes the contribution of the Euler characteristic $\sum(-1)^{i} H_{c, e t, \ell}^{i}\left(S h, \mathcal{F}_{\tau}\right)$ of compact support cohomology to the Euler characteristic $\sum(-1)^{i} H_{\text {et }, \ell}^{i}\left(\overline{S h}, \mathcal{F}_{\tau}\right)$. Since Kottwitz has to work with $\sum(-1)^{i} H_{e t, \ell}^{i}\left(\overline{S h}, \mathcal{F}_{\tau}\right)$ (the fundamental technique used being a comparison between geometric sides of trace formulas), his conjecture looks slightly different to what we have written above. 
(2) Of course, Kottwitz's conjecture has been (partially) proven in many cases of PEL type A or C, and for groups related to inner forms of $G L_{2}$ over a totally real field.

At infinity we have the following:

Theorem 5 (Arthur, [Art] Proposition 9.1). As representations of $\mathbb{C}^{\times}, \mathrm{SL}_{2}(\mathbb{C})$ (i.e., the Hodge structure resp. the Lefschetz decomposition) and $S_{\psi_{\infty}}$ we have

$$
V_{\psi} \cong H_{d R, v}\left(M_{\psi}\right)
$$

for $v$ the place associated to the canonical embedding $E \hookrightarrow \mathbb{C}$.

\section{Remark.}

(1) The way we have described it, it is perhaps not obvious that the above statement makes sense without assuming some conjectures, but it may be formulated entirely without reference to the global $A$-parameter $\psi$ and the motive $M_{\psi}$, using only the local $A$-parameter $\psi_{\infty}$. The definition of $V_{\psi}$ then only depends on $\Pi_{\psi_{\infty}}$, which exists non-conjecturally [VZ], and so does $H_{d R, v}\left(M_{\psi}\right)$ if we define it by the desiderata outlined above, rewritten in a local form (i.e., that the Hodge structure is $\left.\left(\phi_{\infty, r_{L}}|-|^{-d / 2}\right)\right|_{\mathcal{L}_{\bar{E}_{v}}}$ where $\phi_{\infty}$ is $L$-parameter associated with $\psi_{\infty}$; the Lefschetz decomposition is $\left.r_{L} \circ \psi_{\infty}\right|_{\mathrm{SL}_{2}(\mathbb{C})}$, and the action of $S_{\psi_{\infty}}$ is via $r_{L}$ ).

(2) This identifies $V_{\mu}$ and $V_{\psi}$ as vector spaces and hence gives a formula for the dimension of $V_{\psi}$.

The next conjecture is then a consequence of Conjecture 8.1 of [Art], as described above:

Conjecture 6 (Arthur, [Art]). As representations of $\mathbb{C}^{\times}$and $\mathrm{SL}_{2}(\mathbb{C})$ (i.e., the Hodge structure and the Lefschetz decomposition)

$$
W_{d R}^{*}\left(\pi^{\infty}\right)=\operatorname{Hom}_{G(\mathbb{A} \infty)}\left(\pi^{\infty}, H_{(2)}^{*}\left(S h, \mathcal{F}_{\tau}\right)\right)
$$

is isomorphic to

$$
\bigoplus_{\psi: \pi \infty \in \Pi_{\psi} \infty}\left(U_{\pi} \otimes H_{d R, v}\left(M_{\psi}\right)\right)_{\epsilon_{\psi}}
$$

for $v$ the place associated to the canonical embedding $E \hookrightarrow \mathbb{C}$.

We now wish to recast this story using the ideas of Buzzard and Gee and the representation $r_{C}$. Since our $\pi$ are $C$-algebraic, Buzzard and Gee conjecture ([BG] Conjecture 5.3.4) that there exists a Galois representation

$$
\rho_{\pi, \ell}=\rho_{\pi, \ell, \iota}: \operatorname{Gal}(\overline{\mathbb{Q}} / \mathbb{Q}) \rightarrow{ }^{C} G\left(\overline{\mathbb{Q}}_{\ell}\right)
$$

satisfying a list of desiderata, the most important for us being that, for finite primes $p \neq \ell$ such that $\pi$ is unramified, $\left.\rho_{\pi, \ell}\right|_{W_{\mathbb{Q}_{p}}}$ is $\widehat{\widetilde{G}}\left(\overline{\mathbb{Q}}_{\ell}\right)$-conjugate to

$$
w \mapsto \iota\left(\left(\phi_{\pi_{p}}(w),|w|^{1 / 2}\right)\right)
$$

In other words, $\rho_{\pi, \ell}$ is a Galois representation associated with the $L$-algebraic automorphic representation $\widetilde{\pi}$ according to Conjecture 3.2.1 of [BG] (since $w \mapsto\left(\phi_{\pi_{p}}(w)\right.$, $\left.|w|^{1 / 2}\right)$ is the Satake parameter of $\left.\widetilde{\pi}_{p}\right)$. We remark that the set of possible $\rho_{\pi, \ell}$ depends only on $\pi^{\infty}$ and the $L$-packet of $\pi_{\infty}$ (see part 2) of the remark below for some 
remarks on the question of uniqueness). Let $\rho_{E, \pi, \ell}$ denote the restriction of $\rho_{\pi, \ell}$ to $\operatorname{Gal}(\overline{\mathbb{Q}} / E)$. With notation as above, Corollary 3 gives us

Proposition 7. Assume that $M_{\psi}$ exists as above. Then

(1) $H_{e t, \ell}\left(M_{\psi}\right) \cong r_{C} \circ \rho_{\underset{\sim}{\rho, \pi, \ell}}$ as representations of $\operatorname{Gal}(\overline{\mathbb{Q}} / E)$.

(2) $H_{d R, v}\left(M_{\psi}\right) \cong r_{C} \circ \widetilde{\phi}_{\mathbb{C}}$ as representations of $\mathbb{C}^{\times}$.

Remark. (1) Assume Langlands functoriality (say in the weak form of Conjecture 6.1 .1 of $[\mathrm{BG}]$ ). Since $\widetilde{\pi}$ is $L$-algebraic the transfer $\Pi_{E}$ of $\widetilde{\pi}$ to $\mathrm{GL}_{\mathrm{N} / \mathrm{E}}$ (using $r_{C}$ and base change $E / \mathbb{Q}$; here $N=\operatorname{dim} \mathrm{V}_{\mu}$ ) is $L$-algebraic ([BG] Lemma 6.1.2) with $L$-parameter $r_{C} \circ \widetilde{\phi}_{E}$ and can be taken to be isobaric. Thus one sees from Proposition 7 that $M_{\psi}$ is the motive conjecturally associated with $\Pi_{E} \otimes|\cdot|^{(1-N) / 2}$ by Clozel ([Clo] Conjecture 4.5).

(2) $\rho_{\pi, \ell}$ may not be uniquely determined by $\pi$ (see $[\mathrm{BG}]$ Remark 3.2.4 for a discussion and some examples), which forces one to speak of all possible $\rho_{\pi, \ell}$ satisfying the desiderata. Of course, the composition of any such $\rho_{\pi, \ell}$ with a representation of ${ }^{C} G$ does not depend on the choice of $\rho_{\pi, \ell}$ and is determined by $\pi$ at its unramified finite places.

We can now state the minor variations of Conjectures 4 and 6 and Theorem 5 given by Proposition 7:

Conjecture 8. The $\operatorname{Gal}(\overline{\mathbb{Q}} / E)$-representation

$$
W_{e t, \ell}^{*}\left(\pi^{\infty}\right)=\operatorname{Hom}_{G\left(\mathbb{A}^{\infty}\right)}\left(\pi^{\infty}, H_{e t, \ell}^{*}\left(\overline{S h}, \mathcal{F}_{\tau}\right)\right)
$$

is isomorphic to

$$
\bigoplus_{\psi: \pi^{\infty} \in \Pi_{\psi} \infty}\left(U_{\pi} \otimes\left(r_{C} \circ \rho_{E, \pi, \ell}\right)\right)_{\epsilon_{\psi}} .
$$

Theorem 9. We have

$$
V_{\psi} \cong r_{C} \circ \widetilde{\phi}_{\mathbb{C}}
$$

as representations of $\mathbb{C}^{\times}$.

Conjecture 10. As representations of $\mathbb{C}^{\times}$(i.e., Hodge structures)

$$
W_{d R}^{*}\left(\pi^{\infty}\right)=\operatorname{Hom}_{G(\mathbb{A} \infty)}\left(\pi^{\infty}, H_{(2)}^{*}\left(S h, \mathcal{F}_{\tau}\right)\right)
$$

is isomorphic to

$$
\bigoplus_{\psi: \pi^{\infty} \in \Pi_{\psi} \infty}\left(U_{\pi} \otimes\left(r_{C} \circ \widetilde{\phi}_{\mathbb{C}}\right)\right)_{\epsilon_{\psi}} .
$$

Thus, we see that the conjectures of Buzzard and Gee are consistent in a natural way with the known and conjectural properties of the cohomology of Shimura varieties, and offers an explanation for the twist by $-d / 2$.

Remark. We should remark that another possible way of explaining this Tate twist is via the theory of weights for perverse sheaves. Namely, it is natural to consider $\mathcal{F}_{\tau}[d]$ (shift defined by $-[d]^{i}=-{ }^{i+d}$ ) because it is a (pure) perverse sheaf. The shift $[d]$ lowers the weight of the sheaf and its cohomology by $d$, which has the same effect as a Tate twist by $d / 2$ (undoing the Tate twist by $-d / 2$ above) in terms of weights. 


\section{Acknowledgments}

The author would like to thank his PhD supervisors Kevin Buzzard and Toby Gee for asking him the question treated in this paper and for valuable comments on the first draft, Jack Shotton for comments on a later draft, Alexander Paulin for a useful conversation, and the anonymous referee for some useful comments. He also wishes to acknowledge the Engineering and Physical Sciences Research Council for supporting him throughout his doctoral studies. Moreover, it is a pleasure to acknowledge the Fields Institute, where part of the work for this paper was done, for their support and hospitality as well as for the excellent working conditions provided.

\section{References}

[Art] J. Arthur, Unipotent automorphic representations I. Conjectures, Astérisque 171-172 (1989), 13-71.

[BR] D. Blasius and J. Rogawski, Zeta-functions of Shimura varieties, Motives (Seattle, WA, 1991), Proc. Symp. Pure Mathematics 55, AMS, Providence, RI (1994) 525-571.

[Bor] A. Borel, Automorphic L-functions. Automorphic Forms. Representations and L-functions, Proc. Symp. Pure Mathematics 33, Part II, AMS, Providence, RI (1979) 27-61.

[BC] A. Borel and W. Casselman, $L^{2}$-cohomology of locally symmetric manifolds of finite volume, Duke Math. J. 50 (1983), 625-647.

[BG] K.M. Buzzard and T. Gee, The conjectural connections between automorphic representations and Galois representations, To appear in Proc. of the LMS Durham Symp. 2011. Available at http://www2.imperial.ac.uk/ tsg/

[Clo] L. Clozel, Motifs et formes automorphes: applications du principe de fonctorialité. Automorphic forms, Shimura varieties, and L-functions, Proc. of the Ann Arbour Conf., Perspectives on Mathematics 10, Academic Press, Boston, MA (1990), 77-159.

[Del] P. Deligne, Variétés de Shimura : interprétation modulaire, et techniques de construction de modèles canoniques, Automorphic forms, representations and L-functions, Proc. Symp. Pure Mathematics 33, Part II, AMS, Providence, RI (1979), 247-290.

[Kot] R. Kottwitz, Shimura varieties and $\lambda$-adic representations, Automorphic forms, Shimura varieties and L-functions, Proceedings of the Ann Arbour Conf., Perspectives on Mathematics 10, Academic Press, Boston, MA (1990), 161-209.

[Lan] R.P. Langlands, Automorphic representations, motives and Shimura varieties, Ein Märchen. Automorphic Forms. Representations and L-functions. Proc. Symp. Pure Mathematics 33, Part II, AMS, Providence, RI (1979), 205-246.

[Pin] R. Pink, Arithmetical compactification of mixed Shimura varieties, PhD thesis, Bonner Mathematische Schriften, 209, 1989.

[VZ] D. Vogan and G. Zuckerman, Unitary representations with non-zero cohomology, Compos. Math. 53 (1984), 51-90.

Mathematical Institute, University of Oxford, St Giles 24-29, Oxford OX1 3LB, UK E-mail address: johansson@maths.ox.ac.uk 